\title{
Radiation-related polarization instability of Kerr spatial vector solitons
}

\author{
Roman R. Malendevich, Lars Friedrich, and George I. Stegeman \\ Center for Research and Education in Optics and Lasers/School of Optics, University of Central Florida, \\ Orlando, Florida 32816-2700 \\ Jose M. Soto-Crespo \\ Instituto de Óptica, Consejo Superior de Investigaciones Científicas, Serrano 121, 28006 Madrid, Spain
}

Nail N. Akhmediev

Australian Photonics Cooperative Research Centre, Optical Sciences Centre, Research School of Physical Sciences and Engineering, The Australian National University, Canberra ACT 0200, Australia

James Stewart Aitchison

Department of Electronics and Electrical Engineering, University of Glasgow, Glasgow G12 8QQ, Scotland

Received January 18, 2001; revised manuscript received September 7, 2001

\begin{abstract}
We report the experimental observation and numerical simulations of a polarization instability of spatial vector solitons in an AlGaAs slab waveguide. At power levels where the nonlinear index change becomes comparable to the birefringence, the fast soliton becomes unstable. The instability is related to coupling of the fast soliton to the slow radiation modes through phase matching. The combined effects of bifurcation and radiation coupling are the processes ultimately limiting the stability of any single-polarization (fast and slow) Kerr soliton. (C) 2002 Optical Society of America
\end{abstract}

OCIS codes: $060.5530,140.3510$.

\section{INTRODUCTION}

In materials that exhibit the optical Kerr effect, where the nonlinear index change is proportional to the irradiance $\left(\Delta n=n_{2} I\right)$, optical fields localized in space (beams) or time (pulses) can self-trap due to an optically induced positive index change. ${ }^{1}$ In a situation where the nonlinear effect exactly balances the diffraction (in the spatial case) or dispersion (in the temporal case), optical solitons exist, resulting in propagation without change of shape. ${ }^{2}$ Solitons have received a great deal of attention due to their unique physical properties and a number of novel applications, such as long-haul data transmission in optical fibers or light-induced, reconfigurable waveguide structures that become possible. One of the main reasons for the interest in solitons is their remarkable stability, which leads to a particlelike behavior. ${ }^{3,4}$

It is also well known that in the Kerr case a single component soliton is stable only in $(1+1)$-dimensional systems. Typical examples of such systems are fibers, where the two dimensions are the retarded time and the propagation direction, and slab waveguides, where they are the two spatial in-plane dimensions. In $(2+1)$, or higher, dimensional geometries, solitons collapse in Kerr media. ${ }^{5}$ In this paper we deal with planar waveguides, which are essentially a $(1+1)$-dimensional problem; thus the problem of collapse does not appear.

It is remarkable that in the Kerr case the scalar nonlinear problem in $(1+1)$ dimensions is integrable. ${ }^{2}$
Solitons in such systems are nonlinear modes of the nonlinear system, and they do not exchange energy either in collisions with each other or in the process of interaction with radiation. ${ }^{2,4}$ If we perturb the integrable model changing the nonlinearity or take into account birefringence or other physical phenomena, then the system becomes nonintegrable and soliton collisions become inelastic. The solitons now not only exchange energy during collisions but also lose part of their energy into radiation modes. At the same time, it is less known that not only collisions lead to energy losses but even a single soliton may lose energy during propagation due to interaction with radiation modes. This happens due to the possibility of coupling between the soliton and the radiation modes.

A very well known example of such process is soliton propagation in fibers with higher-order dispersion. ${ }^{6}$ In this case, the wave numbers of the linear dispersive waves extend into the region where solitons exist, and due to phase-matching conditions the solitons can interact with radiation and emit small-amplitude linear waves. Another example is the soliton propagation in birefringent media. ${ }^{7}$ The difference between the refractive indices of the two polarization components results in the beating between the two components, and the soliton has slow oscillations that contribute to the phase matching between the soliton and the radiative waves. The energy exchange becomes possible, and as a result the soliton 
loses part of its energy. This process has been numerically investigated in detail in Ref. 7, and analytical calculations for a similar case such as soliton propagation in a nonlinear directional coupler have been done in Ref. 8 . We should note that, even without interaction with radiation, stability properties of vector solitons in birefringent media are quite involved. ${ }^{9-12}$

What is important for us in the present paper is that, due to the interaction with radiation waves, the soliton may become unstable, having an instability growth rate that is usually complex. In the case of third-order dispersion, the radiation phenomena are "beyond all orders" 6 and hardly could be observed experimentally. However, in the case of birefringent media, the perturbation, due to the energy-exchange terms in the propagation equation, is not small at high-enough powers (even if the linear birefringence is small), and radiation may be appreciable, causing the soliton instability, which therefore could be observed experimentally. In the present paper we report, for the first time to our knowledge, an experimental observation of soliton instability, caused by the interaction with radiation modes.

The rest of the paper is organized as follows. In Section 2 we present the theoretical background for beam propagation in Kerr birefringent slab waveguides, in Section 3 we describe our numerical simulations and some numerical results, and in Section 4 we show our experimental results that are then compared with the numerical ones. Finally, Section 5 contains our main conclusions.

\section{THEORETICAL BACKGROUND}

In many cases, soliton propagation can be treated as a scalar problem where the evolution of the field is described by a single nonlinear Schrödinger equation. ${ }^{2}$ In optical fibers, and especially in planar waveguides, this approach must be generalized due to the presence of linear birefringence. In standard fibers there exists a residual birefringence, which is mainly due to small deviations from the cylindrical symmetry and is generally weak. It typically changes with distance in a nondeterministic manner so that it can be treated as a random perturbation of an otherwise isotropic system. For making polarization-preserving fibers it is necessary to introduce artificially asymmetry in the core fiber in order to have well-defined polarization states. For slab waveguides, even those made from isotropic media, there is a relatively large structural birefringence between the TE (polarized in the plane, $x$ ) and TM (dominant polarization orthogonal to the plane, $y$ ) polarized eigenmodes. When the index difference between the high-index film and the bounding media is small (polarization beat length $>0.1 \mathrm{~mm}$ ), the case here, the $z$-polarized field component for TM can be neglected. In this case with well-defined birefringence, the vector nature of the electromagnetic wave needs to be taken into account, and the system has to be described by a set of coupled nonlinear Schrödinger equations for the amplitudes along both birefringent axes. They are $\mathrm{ar}^{12}$

$$
\begin{gathered}
i u_{\xi}-\beta u+\frac{1}{2} u_{\tau \tau}+\left(C|u|^{2}+A|v|^{2}\right) u+B v^{2} u^{*}=0 \\
i v_{\xi}+\beta v+\frac{1}{2} v_{\tau \tau}+\left(|v|^{2}+A|u|^{2}\right) v+B u^{2} v^{*}=0 .
\end{gathered}
$$

Here, the normalized coordinates are $\xi=k z$ and $\tau$ $=k x$, where $z$ is the direction of propagation, $x$ is the transverse coordinate, $k$ is the average propagation constant, and the asterisk denotes complex conjugate. The functions $u$ and $v$ are the normalized amplitudes along both birefringent axes (parallel to $x$ and $y$, respectively),

$$
u=\sqrt{\frac{n_{2}}{d_{\mathrm{eff}} n}} E_{x}, \quad v=\sqrt{\frac{n_{2}}{d_{\mathrm{eff}} n}} E_{y},
$$

$n$ and $n_{2}$ are the linear and nonlinear refractive indices in the medium, and $d_{\text {eff }}$ is the effective height of the slab waveguide, all defined for the appropriate polarization. The normalized birefringence $\beta$ is given by $\beta=\left(k_{y}\right.$ $\left.-k_{x}\right) /(2 k)$, where $k_{x}$ and $k_{y}$ are the waveguide propagation constants for the two modes polarized along $x$ (TE mode) and $y$ (TE mode), respectively.

The Kerr nonlinearity, the magnitude of which is given by $n_{2}$, is further characterized by the parameters $A, B$, and $C$. Here $C$ describes the anisotropy of self-phase modulation, $A$ is the ratio of cross-phase to self-phase modulation, and $B$ is the ratio of four-wave mixing to selfphase modulation [last term in Eqs. (1)]. $B$ couples energy between the two polarizations. The values of the coefficients $A, B$, and $C$ are determined by the characteristics of the medium. In optical fibers, $B=1$ - $A, A=2 / 3$, and $C=1$. For this set of parameters three one-parameter families of solitons exist. In addition to two families of solutions linearly polarized along the fast and slow axes, respectively, a branch of elliptically polarized solitons emerges at power levels above the critical one. ${ }^{9}$ In semiconductor planar waveguides the coefficients have different values, and this leads to more complicated bifurcation patterns. Two additional nontrivial solutions of Eqs. (1) that possess a mixed polarization have been identified. ${ }^{13}$

The polarization instability of the fast soliton in fibers has been discovered numerically in Ref. 14. In particular, the symmetry-breaking soliton instability has been investigated theoretically in Ref. 15 and generalized to the case of solitons in planar waveguides in Ref. 16. However, it was found in Ref. 7 that there are two different physical reasons for the instability of solitons in birefringent media. First, it is the appearance of the modes with mixed polarization that causes the fast (and slow) solitons to be unstable above a certain threshold. Second, even in the absence of the branches with mixed polarizations, fast solitons are unstable due to radiation phenomena. $^{7}$

The reason why the fast solitons are unstable at power levels below the bifurcation point is the coupling to radiation fields. Namely, perturbations of the fast soliton oscillate around zero so that the fast soliton oscillates around its average shape. These oscillations along the propagation direction induce a periodic grating on top of 
the soliton. As a result, phase-matching conditions in the longitudinal direction can be fulfilled, and the soliton couples to orthogonally polarized radiation fields through the grating. As is well known, the phase matching in the transverse direction for narrow sources is not required when the width of the beam is comparable to or smaller than the wavelength. The narrower the beam, the stronger is radiation. In this sense the phenomenon is similar to the surface-emitted second-harmonic generation. In our case, the higher the soliton power, the larger is its angular spectrum because the soliton becomes narrower, and therefore the more likely is its coupling to radiation modes. This instability has a complex exponent in the decay factor, ${ }^{7}$ and its growth rate is the real part of this exponent. Numerical calculations below correspond to this real part.

Experimentally, the instability of the fast temporal soliton in fibers related to the symmetry breaking has been studied in Ref. 17. This instability also occurs in fiber lasers ${ }^{18}$ but in that case it is even more complicated due to the presence of nonconservative effects. Instabilities related to radiation have not been studied experimentally before, either in the temporal or in the spatial case.

Another interesting fact is that, in semiconductor waveguides, symmetry-breaking bifurcations occur from both (TE and TM) branches of orthogonally polarized solitons, with two new eigensolutions emerging, which are el-
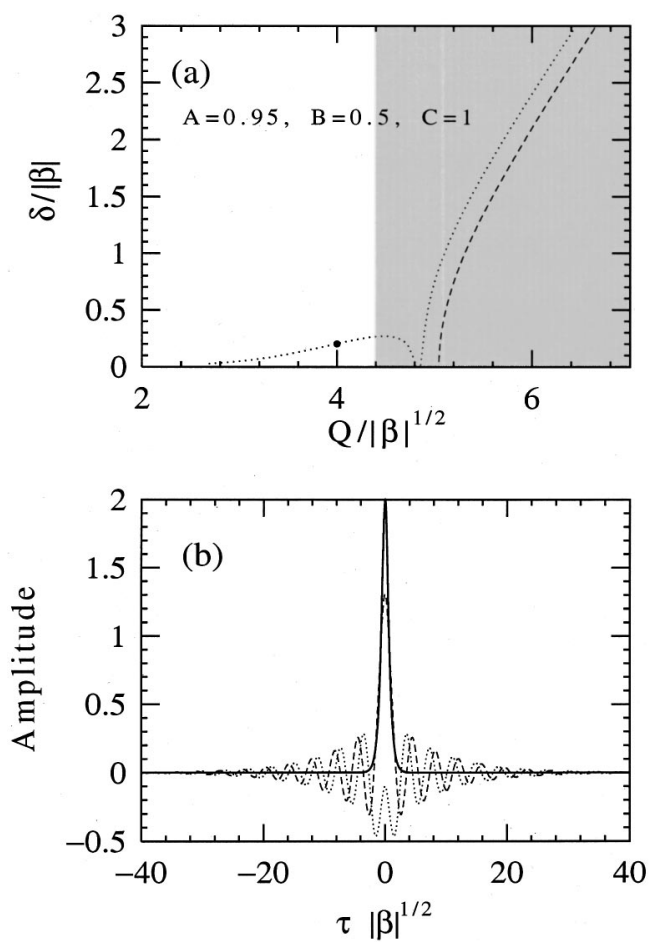

Fig. 1. (a) Growth rate (numerical results) of the instability versus total guided power for TM (dotted curve) or TE (dashed curve) solitons. The power region that is not achievable in the experiment is shown in gray shading. The horizontal axis is given in units of birefringence-normalized power. $Q / \sqrt{|\beta|}=1$ corresponds to a power of $300 \mathrm{~W}$. (b) Real (dotted) and imaginary (dashed curve) parts of the perturbation eigenmode with the largest growth rate associated with the fast soliton (solid curve) for $Q / \sqrt{|\beta|}=4$ (shown by the solid dot in the upper plot). The eigenmode has only the slow component. The solid curve shows the transverse profile of the fast soliton.
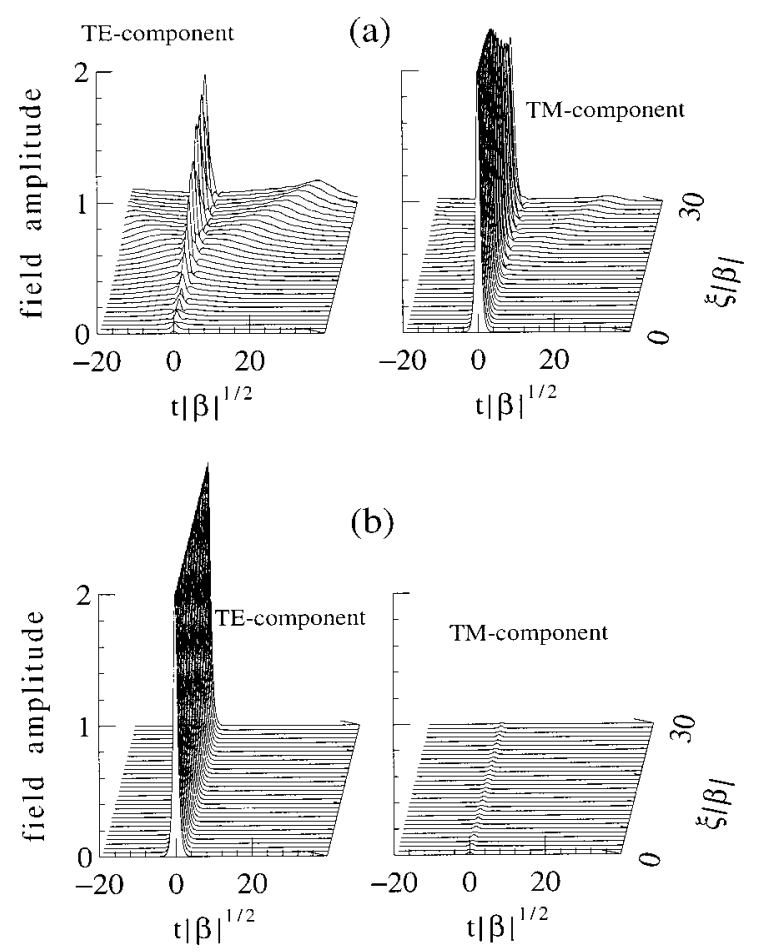

Fig. 2. (a) Beam-propagation-method simulation of the evolution of a fast soliton in the region where the radiation-related instability develops. The emergence of a TE component upon propagation simultaneously with the emission of small dispersive waves is clearly seen. (b) Propagation of the slow soliton. This plot shows that the TE mode is stable. $A=0.95, B$ $=0.5, C=1, Q /|\beta|^{1 / 2}=4$.

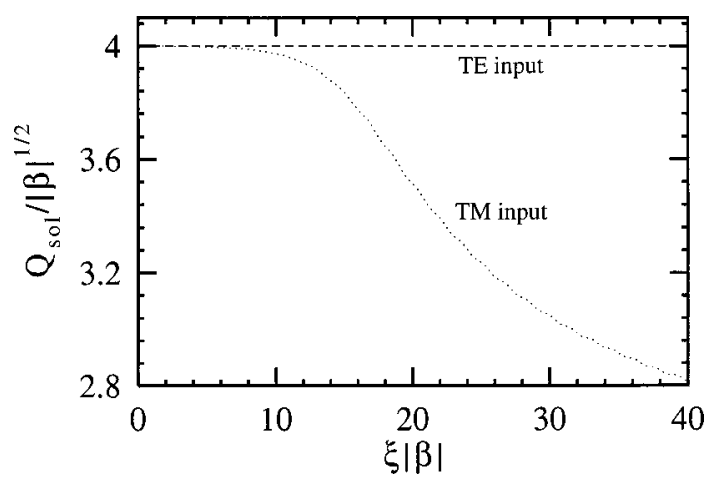

Fig. 3. Amount of power contained in the main beam versus normalized propagation distance.
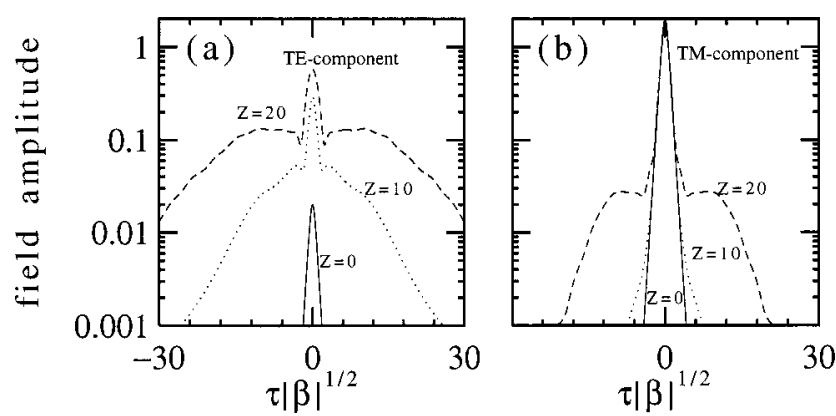

Fig. 4. Beam profiles (in logarithmic scale) during propagation of the fast soliton initially perturbed in accordance with Eq. (6), at $Z=\xi|\beta|=0,10$, and 20. (a) TE-component and (b) TM component. The beam power $Q / \sqrt{|\beta|}=4$. 


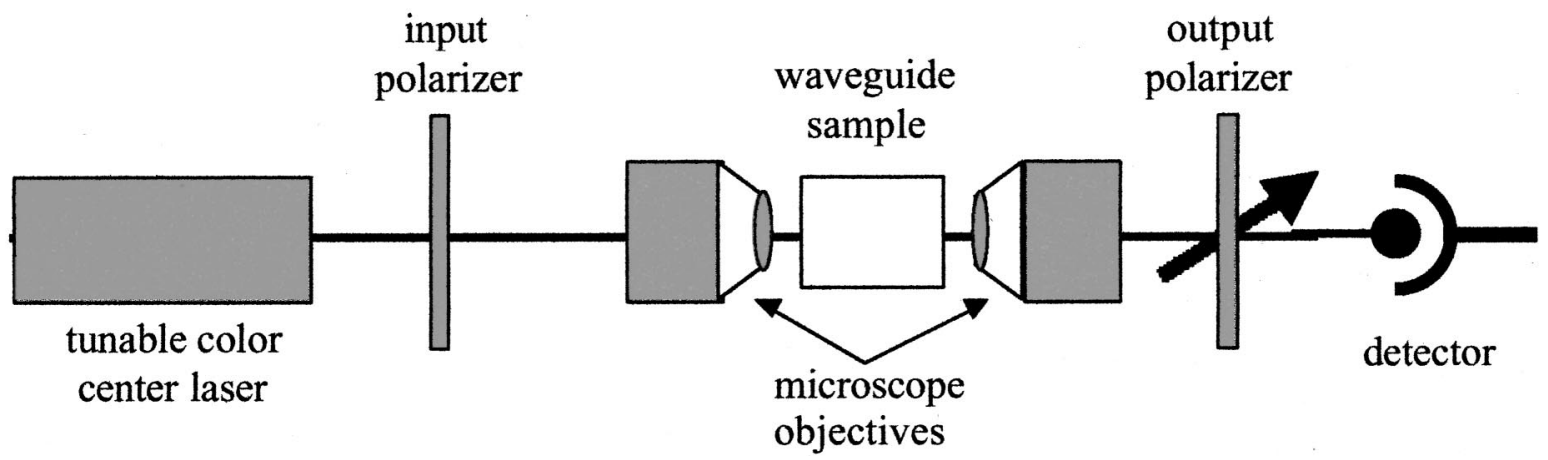

Fig. 5. Schematic of the birefringence measurement. The input polarization was varied by rotating the half-wave plate, and the transmission after the sample with the polarizer at $\theta=45^{\circ}$ was measured for different wavelengths between $1530 \mathrm{~nm}$ and $1630 \mathrm{~nm}$, with a tunable color-center laser as the source.

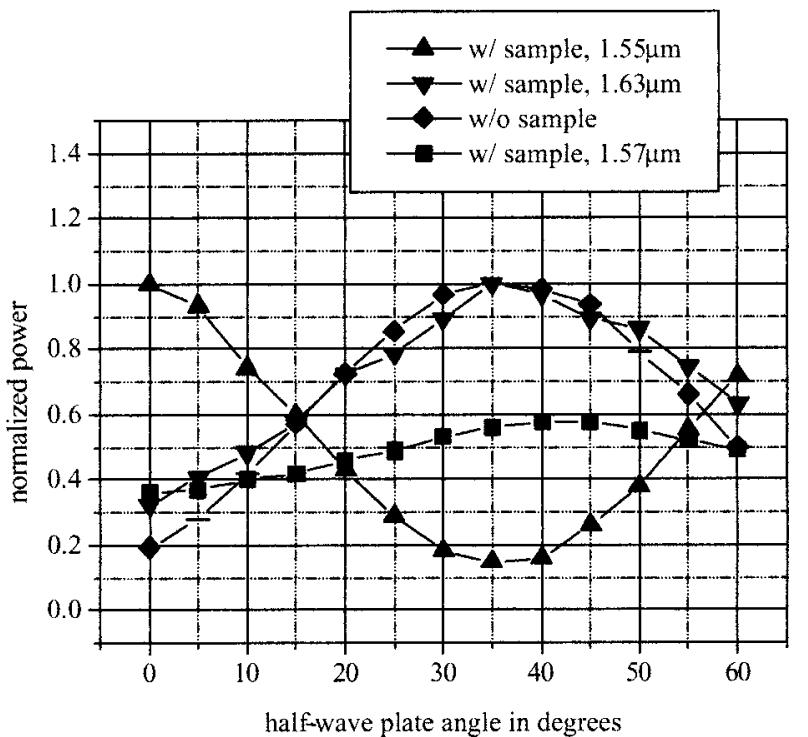

Fig. 6. Transmission measurement with and without the waveguide sample for the birefringence measurement.

liptically and linearly polarized, respectively. ${ }^{19}$ The bifurcation points on these branches are located at different power levels depending on the actual values of the parameters $A, B$, and $C$. In this case, even the slow soliton becomes unstable but at a different (usually higher) threshold power. As we show in Section 3, for the slow soliton, only the symmetry-breaking instability takes place but not the one related to radiation. Hence planar waveguides have soliton features that are absent in fibers, and they therefore deserve special attention. The existence of two new soliton branches has been confirmed theoretically for the particular case of anisotropic cubic media that include semiconductor materials such as GaAs, AlGaAs, or InSb in Ref. 20.

\section{NUMERICAL SIMULATIONS}

Equations (1) can be further simplified with the following rescaling that allows us to eliminate one parameter, $\beta$ :

$$
U=\frac{u}{\sqrt{|\beta|}}, \quad V=\frac{v}{\sqrt{|\beta|}}, \quad Z=|\beta| \xi, \quad X=\tau \sqrt{|\beta|}
$$

With the above change of variables and assuming that $\beta$ is negative, the propagation equations become

$$
\begin{aligned}
i U_{Z}+U+\frac{1}{2} U_{X X} & \\
& +\left(C|U|^{2}+A|V|^{2}\right) U+B V^{2} U^{*}=0, \\
i V_{Z}-V+\frac{1}{2} V_{X X} & \\
& +\left(|V|^{2}+A|U|^{2}\right) V+B U^{2} V^{*}=0 .
\end{aligned}
$$

We study the stability of the solitons linearly polarized along the fast and the slow axes using the linearization method that is analogous to the case of low-birefringent fibers (see details in Ref. 21). Figure 1(a) presents the numerical results obtained from linear-stability analysis. Namely, it shows the largest instability growth rate versus $Q / \sqrt{|\beta|}$, where $Q$ is $Q=\int_{-\infty}^{\infty}\left(|u|^{2}+|v|^{2}\right) d \xi$.

Similar results were obtained for slightly different values of the parameters $A, B$, and $C$. Namely, both types of solitons are unstable just above its corresponding bifurcation point. In addition, the instability related to radiation appears at power levels below the bifurcation point for the fast solitons. Figure 1(b) shows the slow component of the eigenfunction of perturbation with the largest growth rate associated with the fast soliton, which is also plotted in the figure, for $Q / \sqrt{|\beta|}=4$, i.e., in the region below the bifurcation point. The perturbation oscillates around zero and is much broader than the fast soliton, anticipating what will be the actual behavior of these solitons on propagation, i.e., that small dispersive waves will be emitted from the center of the soliton as we show from the numerical solution of Eqs. (4). Note that the eigenfunction of perturbation associated with the fast soliton does not have the fast component.

Figure 2 illustrates the evolution of (a) a fast soliton and (b) a slow soliton slightly perturbed as they propagate along the waveguide. Namely, we used as input the following:

$$
\begin{aligned}
& \text { (a) } U=0.01 V, \quad V=2 \operatorname{sech}(2 X), \\
& \text { (b) } U=2 \operatorname{sech}(2 X), \quad V=0.01 U .
\end{aligned}
$$

Their corresponding energy is in both cases $Q / \sqrt{|\beta|}=4$; thus we are below the bifurcation point. Clearly the slow soliton is stable, whereas the fast one is not. Part of its 
energy is transferred to the TE component, and in this process much of its energy is radiated away. (The pulse profiles are symmetric around $\tau=0$; thus what we observe in the right part of the beam happens also in the left part, which is only partially plotted.) The importance of the radiation emission is clearly demonstrated in Fig. 3, which shows the energy contained in the main beam versus the propagation distance. Initially all the energy is contained in the main beam, and it remains there when the input beam is a slow soliton, but it decreases quickly during propagation if the input is the fast soliton. On the other hand, Fig. 4 shows the beam profiles at different propagation distances, namely, at $Z=0$ (continuous curve), 10 (dotted curve) and 20 (dashed curve) in a $y$-logarithmic scale. In total agreement with the perturbation analysis the slow component acquires a larger pedestal than the fast component that broadens and grows on propagation; i.e., radiation is mainly emitted initially in the slow mode.

In Section 4 we compare the experimental results with numerical ones. In order to have an idea of the correspondence between the physical units and the adimensional ones, let us take typical values $d_{\text {eff }}=1.8$ $\times 10^{-4} \mathrm{~cm}, \quad n_{2}=1.5 \times 10^{-13} \mathrm{~cm}^{2} \mathrm{~W}, \quad$ and $\beta=-8.5$ $\times 10^{-5}$. Then, for $\lambda=1.57$ we get for the power

$$
P=\frac{d_{\mathrm{eff}} \lambda}{2 \pi n_{2}} Q \approx 29984 Q W ;
$$

thus $Q / \sqrt{|\beta|}=1$ corresponds to a power $P \approx 300 \mathrm{~W}$, a sample length of $1.5 \mathrm{~cm}$ corresponds to $Z_{L}=|\beta| \xi_{L} \approx 17$, and a beam width of $1.8 \mu \mathrm{m}$ corresponds to $X_{w}=\sqrt{|\beta|} \tau_{w}$ $\approx 1.8$.

\section{EXPERIMENTAL RESULTS}

Experimentally, the principal problem in studying instabilities in Kerr slab waveguides is to identify a material system in which the response is truly Kerr. In addition, the birefringence should be small enough and the Kerr coefficient large enough that instabilities will occur before the damage threshold is reached. In our experiment we used the half-bandgap nonlinearity of AlGaAs. The samples were grown by molecular-beam epitaxy on a GaAs substrate. The refractive-index tuning required for defining the waveguide structure was achieved by changing the $\mathrm{Al} / \mathrm{Ga}$ ratio.

The lower cladding layer was $4 \mu \mathrm{m}$ thick and consisted of $\mathrm{Al}_{0.24} \mathrm{Ga}_{0.76} \mathrm{As} \quad(n=3.325$ at $1550 \mathrm{~nm})$. The highindex waveguide core was $1.5 \mu \mathrm{m}$ thick and consisted of $\mathrm{Al}_{0.18} \mathrm{Ga}_{0.82} \mathrm{As}$. Finally, the upper cladding was a $1.5-\mu \mathrm{m}-$ thick layer of $\mathrm{Al}_{0.24} \mathrm{Ga}_{0.76} \mathrm{As} \quad(n=3.336$ at $1550 \mathrm{~nm})$. Slab waveguides, $15 \mathrm{~mm}$ long, were cleaved from the wafer. The effective thickness of the resulting waveguide

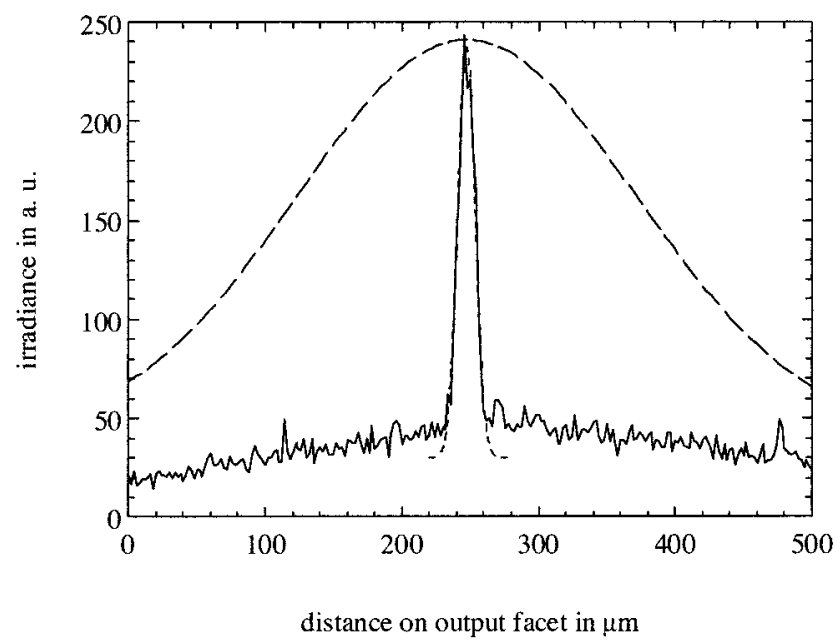

Fig. 8. Horizontal line scans of the near-field image of the soliton showing the input beam (dots), the shape of a diffracted lowpower beam (dashed curve), and the soliton (solid curve) at the end of the sample.

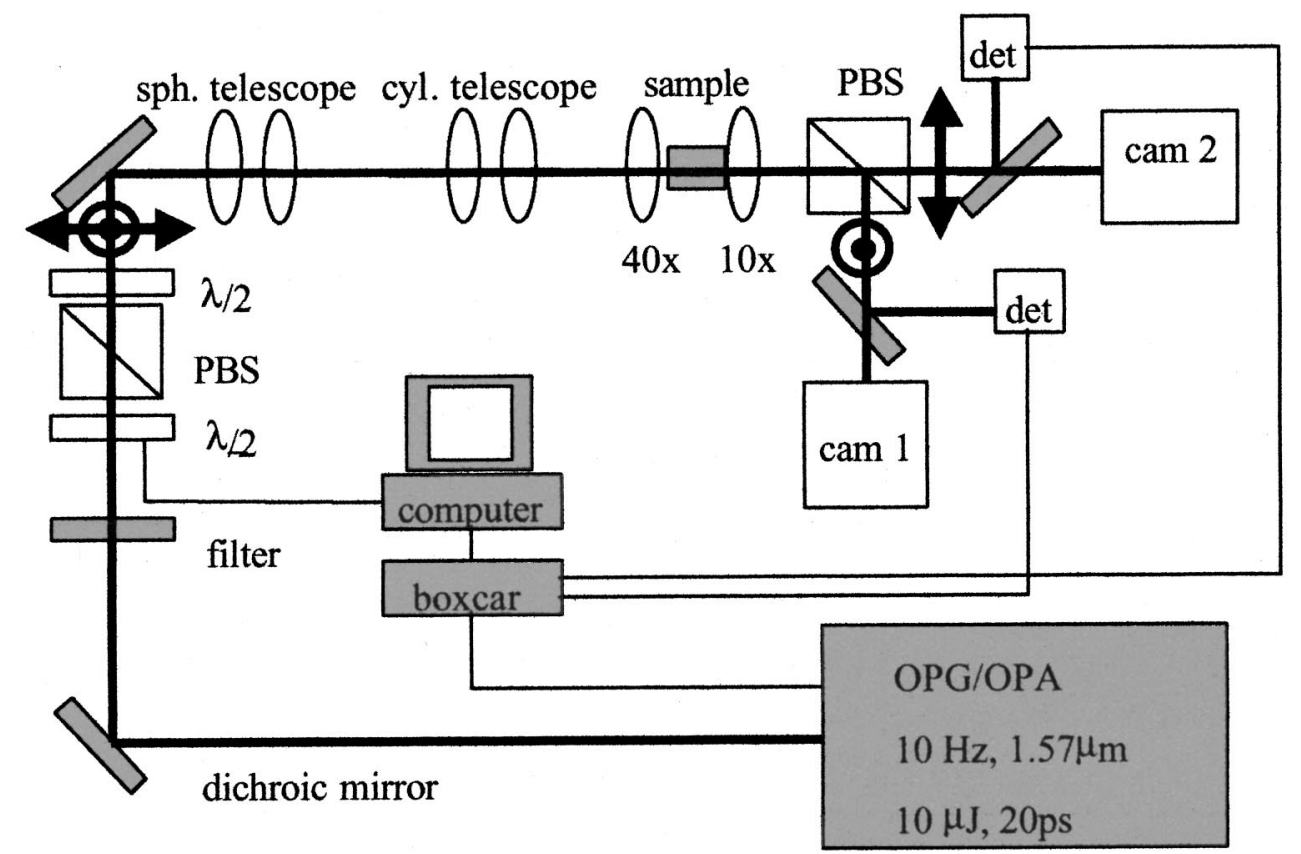

Fig. 7. Experimental setup: PBS, polarizing beam splitter; $\lambda / 2$, half-wave plate; det, detector; cam, camera. 
was $1.8 \mu \mathrm{m}$. From previous measurements in similar samples, we found that in the 1.55- $\mu \mathrm{m}$-wavelength region the nonlinear response is effectively Kerrlike (for photon energies just below one half of the semiconductor bandgap), with $n_{2}=1.5 \times 10^{-13} \mathrm{~cm}^{2} / \mathrm{W}, A \approx 1, B \approx 0.5$, and $C \approx 11^{17}$ For the given waveguide structure, this results in $n_{2 \text { eff }}=1.5 \times 10^{-13} \mathrm{~cm}^{2} / \mathrm{W}$. In such samples, singlepolarization, Manakovlike, and vector solitons have all been observed under appropriate conditions. ${ }^{19,20,22}$ The typical birefringence of such samples was $>10^{-3}$, which results in the critical power required for the observation of instabilities approaching the damage threshold of the samples.

Due to its cubic crystal symmetry, bulk AlGaAs exhibits no material birefringence. In molecular-beam-epitaxy grown AlGaAs waveguides such as the one used for this experiment, the value of the birefringence has been known to differ considerably from the structural waveguide birefringence due to stress induced by the growth process. The effects of the instability scale with the value of the waveguide birefringence; therefore a measurement of this parameter is crucial for the evaluation of the experimental results.

We measured the birefringence by launching a linearly polarized low-power input beam with a varying angle of the launch polarization to the TE axis and by analyzing the polarization state at the waveguide output as a function of wavelength. The setup is shown schematically in Fig. 5. A continuously tunable color-center laser (Burleigh FCL-120) was used as a light source, and the input polarization was varied by use of a half-wave plate. At the waveguide output, an analyzer at $45^{\circ}$ to both the TE and TM axes was used in front of a detector. Special care was taken so that the only wavelength dependence of the experiment arose from the sample itself.

The birefringence-induced phase delay $\Gamma$ between the TE and TM depends on the sample length $L$, the birefringence $n_{x}-n_{y}$, and the wavelength $\lambda$ :

$$
\Gamma=\frac{2 \pi}{\lambda}\left(n_{x}-n_{y}\right) L
$$

The different values of $\Gamma$ result in varying transmission as a function of the angle of the input polarization with the wavelength axes. Figure 6 shows the transmission as a function of half-wave-plate angle $\Omega$ for two wavelengths, with and without the sample. Measurements were performed at a number of wavelengths in order to avoid any ambiguity in the value of $\Gamma$ with respect to multiples of $2 \pi$. Neglecting the wavelength dispersion of the birefringence, we found $n_{x}-n_{y}=5.5 \pm 1.0 \times 10^{-4}$, which is considerably higher than the calculated structural birefringence for this waveguide configuration $\left(n_{x}-n_{y}\right.$ $\left.=1.5 \times 10^{-4}\right)$. This yields $\beta=-8.5 \times 10^{-5}$. The experiments for the polarization instability were performed with a lithium triborate based optical parametric generation/amplification system with a $10-\mathrm{Hz}$ repetition rate and an idler wavelength of $1.57 \mu \mathrm{m}$, pumped at 0.532 $\mu \mathrm{m}$ with a doubled Nd:YAG laser with a subsequent regenerative amplifier. Pulse lengths were of the order of $20 \pm 5 \mathrm{ps}$, with up to $1.2 \mu \mathrm{J}$ of energy. The system oper- ated below the saturation point for the parametric process and had a shot-to-shot rms stability of $\pm 30 \%$.

The experimental setup is shown in Fig. 7. The launch power was varied with a half-wave-plate/polarizer combination, which was operated by a stepper motor. A second half-wave plate was used in order to control the polarization state with which the soliton was launched. The crossed-polarizer transmission was $2 \times 10^{-4}$. However, with the sample present in the setup, the best extinction ratio decreases to $2 \times 10^{-3}$ for both input polarizations due to waveguide imperfections so that, during launch and propagation, $0.2 \%$ appears in the crossed polarization. This acts as a seed for the polarization-conversion process. The beam was then resized, and a cylindrical telescope was used to form an elliptical in-coupling spot, thus launching solitons $\sim 12 \mu \mathrm{m}$ wide (ellipticity 1:8). For solitons of width $12 \mu \mathrm{m}$, the present sample is roughly ten diffraction lengths long, and the soliton peak power is $\approx 500 \mathrm{~W}$. The beam was coupled into the guide with a $40 \times$ microscope objective. The output was imaged by a $10 \times$ microscope objective; TE and TM components were separated by a second polarizing beam splitter and imaged onto a camera, or a detector. Each detector was calibrated and read out with boxcar integrators, and the signal was averaged over 10 pulses. In Fig. 8, a horizon-
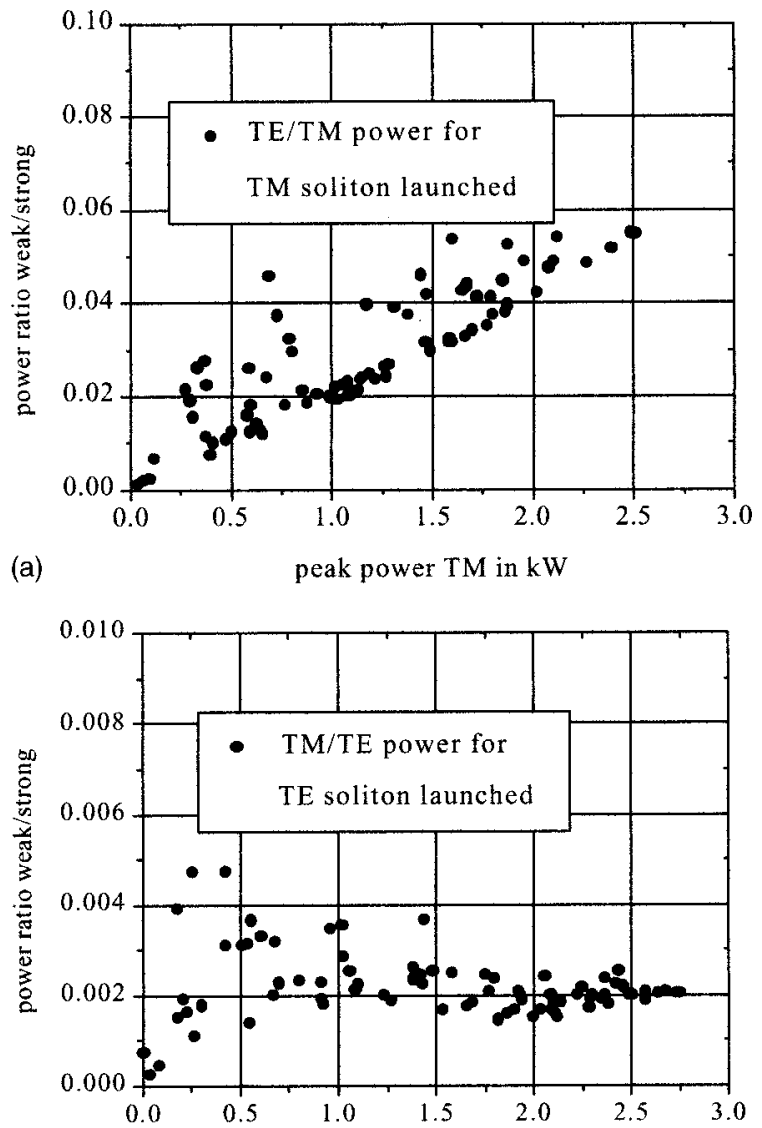

(b)

peak power TE in $\mathrm{kW}$

Fig. 9. Experimental irradiance ratio of weak to strong polarization versus total guided power for a beam launched into the waveguide polarized along the (a) $x$ axis (TE component) or (b) $y$ axis (TM mode). 


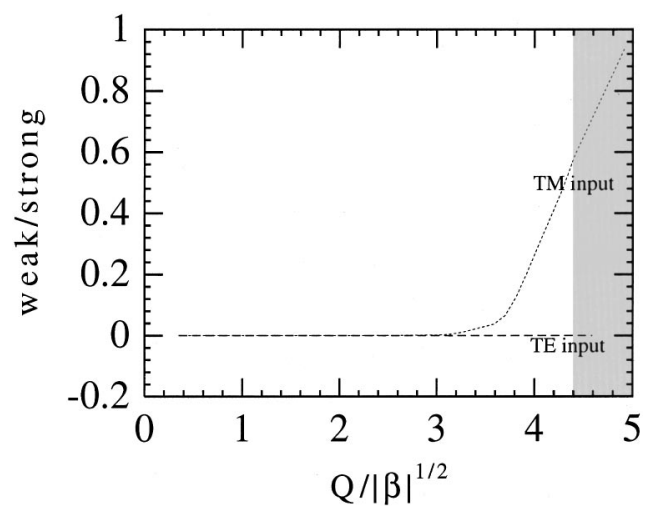

Fig. 10. Theoretical irradiance ratio of weak to strong polarization versus total guided power for a TM (dotted curve) and TE (dashed curve) Gaussian beam launched into the waveguide. The power region that is not achievable in the experiment is shown in gray.

tal line scan of the input spot and the soliton at the end of the waveguide is shown, together with the theoretical curve for the (diffracted) low-power beam at the end of the sample. Also, an image of the soliton near field at the waveguide end face is shown.

In order to investigate the stability of vector solitons in this system, we launched solitons that were linearly polarized along the $x$ and $y$ axes, respectively (i.e., TE and TM polarized) by selecting the proper angle for the halfwave plate before the polarizer. We then changed the power launched (keeping the beam width at $12 \mu \mathrm{m}$ ) by rotating the half-wave plate before the polarizer. Note that although input beams with powers below or above $500 \mathrm{~W}$ do not couple directly at the input to solitons, they generate solitons after some propagation by trade-offs between the peak power and the width. ${ }^{23}$ With no polarization instability present, the fraction of the output light in the polarization orthogonal to the one launched (referred as the "weak" polarization as opposed to the "strong" polarization launched) should be constant. It cannot, however, be expected to be zero due to a finite amount of leakage through the polarizer and stray scattering. Prior to using this setup for actual measurements, we carefully calibrated the measurement system in order to ensure that there was no power dependence in the transmission of either polarization.

We took images of the soliton launched into the waveguide with an IR-sensitive camera and a frame grabber. Figures 9(a) and 9(b) show our results for TM and TE solitons launched respectively: At low powers, there is considerable uncertainty in both the weak and the strong signals due to the low signal levels and consequently the ratio of weak/strong fluctuates strongly at low powers. At higher power levels, for the slow mode (TE), the relative amount of light polarized orthogonal to the launch direction is constant, independent of launched power. For the fast mode (TM), an increasing amount of light is coupled to the orthogonal polarization with increasing input power, indicating an instability of this mode.

Figure 10 shows the numerical results for the simulation of the above experiment for cw input beams. There is good qualitative agreement with the results of the ex- periment, which show that the TE mode is stable and the TM mode unstable. We did not perform a detailed analysis of the data and direct comparison with theory because of the large uncertainties in the laser's shot-to-shot peak power and in the birefringence. The waveguide-coupling efficiency makes the exact location of our experiments in Fig. 1 unknown to a factor of at least 50\% and because for most of the power range investigated the pulsed beams launched have to first evolve into solitons with propagation distance. However, it is noteworthy that the anomalous distribution in the noise in the data for TE/TM, i.e., always to the high conversion side, is characteristic of exponential-gain processes in which a small increase in power produces greatly increased polarization conversion.

\section{CONCLUSIONS}

We have experimentally observed and theoretically analyzed the radiation-related polarization instability of spatial vector solitons in Kerr media.

\section{ACKNOWLEDGMENTS}

This research was supported at the Center for Research and Education in Optics and Lasers, by the National Science Foundation and a U.S. Army Research Office Mulidisciplinary University Research Initiative, at the University of Glasglow by the Engineering and Physical Science Research Council, and at the Instituto de Optica of the Consejo Superior de Investigaciones Científicas by the Dirección General de Enseñanza Superior under contract BFM2000-0806. N. Akhmediev acknowledges support from the U.S. Army Research Office, Far East (grant N62649-01-1-0002).

\section{REFERENCES}

1. R. Y. Chiao, E. Garmire, and C. H. Townes, "Self-trapping of optical beams," Phys. Rev. Lett. 13, 479-482 (1964).

2. V. E. Zakharov and A. B. Shabat, "Exact theory of two dimensional self focusing and one dimensional self modulation of nonlinear waves in nonlinear media," Zh. Eksp. Teor. Fiz. 61, 118-127 (1971) [Sov. Phys. JETP 34, 62-69 (1972)].

3. C. R. Menyuk, "Soliton robustness in optical fibers," J. Opt. Soc. Am. B 10, 1585-1591 (1993).

4. N. J. Zabusky and M. D. Kruskal, "Interaction of solitons in a collisionless plasma and the recurrence of initial states," Phys. Rev. Lett. 15, 240-243 (1965).

5. V. N. Vlasov, I. A. Petrishev, V. I. Talanov, and Izv. Vuzov, Radiofizika 14, 1353 (1971); J. J. Rasmussen and K. Ripdal, "Blow-up in nonlinear Schrödinger equations. I. A general review," Phys. Scr. 33, 481-497 (1986).

6. P. K. A. Wai, H. H. Chen, and Y. C. Lee, "Radiation by 'solitons' at zero group-dispersion wavelength of single-mode optical fibers," Phys. Rev. A 41, 426-439 (1990).

7. N. N. Akhmediev and J. M. Soto-Crespo, "Dynamics of solitonlike pulse propagation in birefringent optical fibers," Phys. Rev. E 49, 5742-5754 (1994).

8. A. V. Buryak and N. N. Akhmediev, "Influence of radiation on soliton dynamics in nonlinear fibre couplers," Opt. Commun. 110, 287-292 (1994).

9. N. N. Akhmediev, A. Buryak, and J. M. Soto-Crespo, "Elliptically polarized solitons in birefringent optical fibers," Opt. Commun. 112, 278-282 (1994).

10. W. Wang, R. Barille, and G. Rivoire, "Influence of soliton propagation on the beam-polarization dynamics in a planar waveguide," J. Opt. Soc. Am. B 15, 2731-2737 (1998). 
11. Y. Chen, "Stability criterion of coupled soliton states," Phys. Rev. E 57, 3542-3550 (1998).

12. D. C. Hutchings, J. M. Arnold, and D. F. Parker, "Stationary mixed-polarization spatial solitons and their stability in semiconductor waveguides," Phys. Rev. E 58, 6649-6658 (1998).

13. E. A. Ostrovskaja, N. N. Akhmediev, G. I. Stegeman, J. U. Kang, and J. S. Aitchison, "Mixed-mode spatial solitons in semiconductor waveguides," J. Opt. Soc. Am. B 14, 880-887 (1997).

14. K. J. Blow, N. J. Doran, and D. Wood, "Polarization instabilities for solitons in birefringent fibers," Opt. Lett. 12, 202-204 (1987).

15. E. M. Wright, G. I. Stegeman, and S. Wabnitz, "Solitarywave decay and symmetrybreaking instabilities in twomode fibers," Phys. Rev. A 40, 4455-4466 (1989).

16. C. M. De Sterke and J. E. Sipe, "Polarization instability in a waveguide geometry," Opt. Lett. 16, 202-204 (1991).

17. Y. Barad and Y. Silberberg, "Polarization evolution and polarization instability of solitons in a birefringent optical fiber," Phys. Rev. Lett. 78, 3290-3293 (1997).

18. S. T. Cundiff, B. C. Collings, N. N. Akhmediev, J. M. Soto-
Crespo, K. Bergman, and W. H. Knox, "Observation of polarization-locked vector solitons in optical fiber," Phys. Rev. Lett. 82, 3988-3991 (1999).

19. J. U. Kang, G. I. Stegeman, J. S. Aitchison, and N. N. Akhmediev, "Observation of Manakov spatial solitons in AlGaAs planar waveguides," Phys. Rev. Lett. 76, 36993702 (1996).

20. D. C. Hutchings, J. M. Arnold, and J. S. Aitchison, "Theory of mixed-polarization propagation in anisotropic cubic media," Opt. Quantum Electron. 30, 771-782 (1998).

21. N. N. Akhmediev, A. V. Buryak, J. M. Soto-Crespo, and D. R. Andersen, "Phase-locked stationary soliton states in birefringent nonlinear optical fibers," J. Opt. Soc. Am. B 12, 434-439 (1995).

22. J. S. Aitchison, D. C. Hutchings, J. U. Kang, G. I. Stegeman, E. Ostrovskaya, and N. Akhmediev, "Powerdependent polarization dynamics of mixed-mode spatial solitary waves in AlGaAs waveguides," J. Opt. Soc. Am. B 14, 3032-3037 (1997).

23. For completely equivalent discussion for temporal soliton, see G. P. Agrawal, Nonlinear Fiber Optics, 2nd ed. (Academic, San Diego, 1995). 